TO: $\quad$ A/Administrator

FROM: $\quad$ S/Associate Administrator for Space Science

SUBJECT: Post-Launch Mission Operation Report (MOR) for the Hubble Space Telescope First Servicing Mission

The first planned mission to service the Hubble Space Telescope (HST) in-orbit, designated STS-61, was launched on December 2, 1993, and successfully completed its servicing assignment on December 10, 1993. Subsequently, the maintenance and replacement activities performed on the telescope were verified successful under control of the Space Telescope Operations Control Center at GSFC and the Space Telescope Science Institute at Johns Hopkins University. The enclosed Post-Launch MOR is herein submitted as required by HQMI 8610.1C.

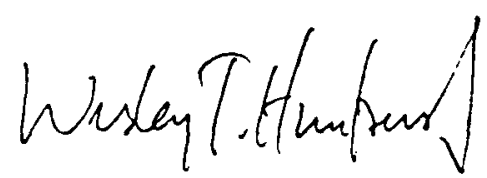

Wesley T. Huntress, Jr.

Enclosure

ICH (JOP H1 51)
HISTORY OFFICE




\section{Post Launch}

Mission Operation Report

OFFICE OF SPACE SCIENCE

Report No. S 458-61-93-02

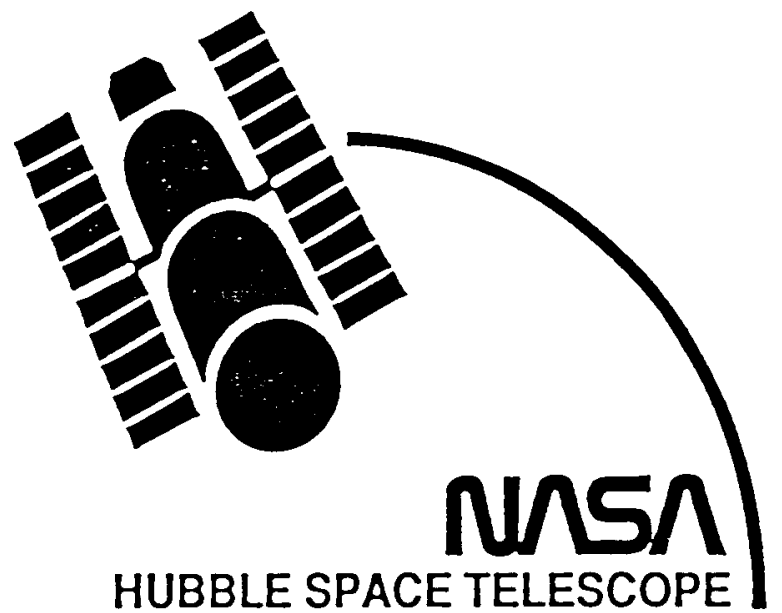

Hubble Space Telescope-First Servicing Mission

April 1994 


\section{Table of Contents}

Foreword i

$\begin{array}{ll}\text { Background } & 1\end{array}$

Mission Support $\quad 2$

Pre-Launch Mission Objectives and Success Criteria 3

HST/STS-61 Mission Assessment Statement 6 


\section{FOREWQRD}

MISSION OPERATION REPORTS are published expressly for the use of NASA senior management. The purpose of these reports is to provide NASA senior management with timely, complete, and definitive information on flight mission plans and to establish official mission objectives which provide the basis for assessment of mission accomplishments.

Reports are prepared and issued for each flight project just prior to launch. Following launch, updated reports for each mission are issued to keep management currently informed of definitive mission results as provided in NASA Management Instruction HQ NMI 8610.1C.

These reports are sometimes highly technical and are for personnel having program/project management responsibilities. The Public Affairs Division publishes a comprehensive series of reports on NASA flight missions which are available for dissemination to the news media. 


\section{BACKGROUND}

The Hubble Space Telescope (HST) is a high-performance astronomical telescope designed to operate in low-Earth orbit. It is approximately 43 feet long with a diameter of 10 feet at the forward end and 14 feet at the aft end. Weight at launch was approximately 25,000 pounds. In principle, it is no different than the reflecting telescopes in ground-based observatories. Like ground-based telescopes, HST was designed as a general-purpose instrument capable of using a wide variety of scientific instruments at its focal plane. The telescope's planned useful operational lifetime is 15 years during which it will make observations in the ultraviolet, visible, and infrared portions of the spectrum. This extended operational life of HST will be made possible by using the capabilities of the Space Transportation System to periodically visit the orbiting HST to replace failed or degraded components, install instruments with improved capabilities, reboost HST to higher altitudes when required to compensate for gradual orbit decay, and bring HST back to Earth when its useful lifetime is completed.

HST was launched into a 330 nautical mile orbit on April 24, 1990, and after a period of subsystem and scientific instrument checkout and calibration, began taking scientific observations. Shortly thereafter, scientists and engineers discovered two distinct phenomenon that degraded the observed scientific data. First, it was determined that HST's primary mirror exhibited spherical aberration. This flaw prevented light from being focused at a single point, denying the scientific observers the total clarity and scientific detail they were seeking. The second problem involved the electrical power-producing solar arrays. When HST passed in and out of orbital shadow, the thermal expansion and contraction from the heating and cooling of the arrays caused the arrays to undergo a transient distortion, which induced a jitter strong enough for the HST's pointing and control system to lose lock on target stars.

A planned servicing mission, already scheduled for 1993, was then refocused on restoring HST to its planned optical capability and repairing other subsystems that failed with time. Corrective optics for the instruments were designed and developed. Upgraded solar arrays were developed and delivered to GSFC by the European Space Agency. Replacement or augmentation hardware for failed or degraded components were designed, manufactured, and tested in high fidelity mechanical and electrical simulators at GSFC. The Shuttle mission was manifested and mission-planning coordination between GSFC, JSC, and KSC intensified. A series of periodic joint working group meetings hammered out the many technical details. The STS-61 flight crew was named and underwent extensive training in simulators and neutral buoyancy water tanks. Procedures and flight rules were written to cover both normal and abnormal situations. Tools and crew aids to assist the astronauts in Extra Vehicular Activity (EVA) tasks, and carriers to house the replacement hardware, were developed and tested. Ground flight controllers at JSC and GSFC were selected and trained. They participated in many simulations designed to replicate mission environments and conditions. 


\section{MISSION SUPPORT}

The long and intense training period for flight and ground crews ended during the early morning hours of December 2, 1993 (after a one day-delay due to high wind conditions), when the Space Shuttle Endeavour carrying a crew of seven astronauts and the HST replacement hardware lifted off from KSC to rendezvous with HST. After successfully grappling and docking HST in the Shuttle payload bay, astronaut teams performed precision repair and replacement tasks during 5 days of EVA. They were assisted in their tasks by operations flight controllers at JSC and GSFC. The Shuttle flight was controlled from the JSC Mission Control Center where the normal complement of Shuttle flight controllers were assisted by EVA and Payloadtrained specialists. GSFC Project Management personnel, led by the Associate Director of Flight Projects for HST, and NASA Headquarters Program Office management were available 24 hours a day in the JSC Customer Support Room to assist in any flight decision process that was not covered by flight mission rules.

The flight control team for HST operations was divided between JSC and GSFC. Coordination between the two for servicing actions was accomplished by the GSFC Mission Manager located at JSC. Assisting the Mission Manager were HST system engineers, flight support systems and scientific instrument personnel, and EVA representatives. At GSFC, teams of engineers and scientists located in the Space Telescope Operations Control Center provided operational control of HST until it was docked with the Endeavour. These personnel conducted real-time commanding of HST to safe systems prior to EVAs, accomplished aliveness and functional tests on in-flight serviced hardware, and provided troubleshooting expertise when required.

The entire mission was a precise orchestration of flight crew and ground actions which was governed by a pre-launch, jointly developed, integrated timeline. This timeline was so well planned that only minor amendments were required in near real-time to reflect current conditions. Coordination between the two Centers was outstanding and conducted in a very professional manner. This close teamwork contributed immensely to the success of the servicing mission.

After five long days of EVA, during which all of the planned servicing tasks were completed by the astronauts, the HST appendages were extended and the repaired telescope was redeployed into its orbit. Once released from the Endeavour, the HST command and control reverted solely to GSFC, and a three and one-half month period of Observatory Verification was begun. During this time, systems and subsystems were checked out. Scientific instruments were aligned to HST's optical axis and calibrated to the new characteristics provided by the recently installed corrective optics. This verification period, which has been completed, proved the success of the repair and replacement actions performed during the First Servicing Mission. 


\section{PRE LAUNCH MISSION OBIECTIVES AND SUCCESS CRITERIA}

Before launch of STS-61, a list of mission objectives were generated and the mission success criteria were published in the pre-launch Mission Operation Report. These objectives are summarized below:

\section{Objectives}

Space Shuttle mission STS-61 was the first of several planned servicing missions for HST, intended to periodically replace failed components and upgrade scientific instruments with improved versions to keep the telescope viable and productive throughout its planned 15-year lifetime. This First Servicing Mission was also intended to correct several design flaws that were detected shortly after the launch of HST. There were three overall mission objectives for the STS-61 repair mission:

\section{- To Restore the Planned Scientific Capabilities}

One complexity of the First Servicing Mission was the necessity for adding optical elements in the light path to correct the spherical aberration. These corrective optics were required to provide the quantitative science capability to enable key scientific programs to be carried out as originally planned. The addition of the COSTAR and the installation of WFPC2 both contributed to recovering these capabilities.

- To Restore the Reliability of Vehicle Systems

Failed or degraded components had depleted some of the original subsystem redundancy, which had to be restored to allow continued science operations until the next servicing mission in 1997. Anomalous components that required servicing included the solar arrays, gyroscope sensing units, gyroscope electronics, magnetometers, solar array drive electronics, and electrical fuses.

- To Validate the On-Orbit Servicing Concept for HST

Validation of the concept of on-orbit servicing as the way to achieve HST's full 15-year life was required to provide a foundation for future servicing missions

\section{Mission Success Criteria}

Given the expressed mission objectives and the fact that the First Servicing Mission was to be a newly attempted, complicated set of tasks, it was necessary to consider what had to be achieved to constitute mission success.

To achieve the highest state of HST operational capability, the STS-61 mission planning strategy was to prepare for servicing options that would accomplish more than the required primary servicing tasks and to take along the associated HST flight hardware to install if time permitted. All servicing tasks were prioritized and 
planned for accomplishment in priority order, consistent with timeline and Shuttle servicing constraints. Lower priority items were to be achieved on a best-efforts, time-available basis. If servicing activities were completed faster than planned, the extra time available was to be used for lower priority tasks. Appropriate flight rules were developed to govern the rescheduling of tasks in real time as necessary to respond to changing in-flight situations.

With these considerations in mind, two levels of mission success were defined. The accomplishments necessary to minimally satisfy the overall mission objectives were those that would leave HST with:

- at least 3 reliable (newer design) gyro systems, and

- either an operational WFPC2 or COSTAR.

This level of accomplishment would correct the optics for part of the science instrument complement, restore the most critical vehicle redundancy, and demonstrate on-orbit servicing theory. This goal provided a reasonable probability that the telescope would continue to carry out some of the key programs until another Shuttle flight could be launched to complete the repairs.

In order to be considered fully successful, the primary list of servicing tasks had to be accomplished. This primary list consisted of:

- Solar Array II

- Gyro Pair \#2

- WFPC2 and instrument fuses

- costar

- Magnetometer System \#1

- Gyro Pair \#3 (with electronics unit \#3)

- Solar Array Drive Electronics \#1

The prominent position of the replacement solar arrays on the priority list assumed that the solar array I blankets would be fully retracted into their cassettes. If for any reason they were intentionally not retracted and servicing instead conducted with solar array I still deployed, the installation of solar array II would not have been considered a primary servicing task. This is the reason solar array replacement did not appear in the minimum success criteria scenario.

It should also be noted that on-board gyro pair \#1 (which was not replaced) consists of the newer-design gyro units, such that replacement of either pair \#2 or pair \#3 would have satisfied the minimum success criteria. Although preliminary planning for a contingent backup servicing shuttle mission if all primary tasks had not been accomplished was done, these plans were not needed due to the complete success of the servicing tasks on STS-61. 
The completion of the entire primary servicing task list provided an excellent chance of maintaining HST quantitative science operations until the scheduled 1997 servicing mission. The remaining items on the manifest were secondary servicing tasks that were to be done as time permitted. This list consisted of:

- GHRS Redundancy Kit

- DF-224's 386 co-processor

- Magnetometer system \#2

- Gyro fuse plugs, and

- Electronics Control Unit for gyro pair \#1

Although accomplishment of these items was to provide additional HST system redundancy and enhanced operational capability, they were not considered essential for mission success (based on the pre-servicing mission state of the HST vehicle) and would have been deferred until the 1997 servicing mission, if they had not been completed on STS-61. 


\section{HST/STS-61 MISSION ASSESSMENT STATEMENT}

In December 1993, the Space Shuttle Endeavour was launched, rendezvoused, and docked with the Hubble Space Telescope. Change-out or installation of all items on both the primary and secondary servicing task lists was accomplished by the astronauts during 5 days of intensive EVA activities. Thus, not only were all three of the pre-launch mission objectives met, but the secondary objectives were accomplished also. Scientific capabilities have been restored. The optical corrections have proven to be outstanding as evidenced by received post-mission images. Vehicle systems were restored to a fully redundant status. The post-mission period of Observatory Verification, where systems were tested and scientific instruments aligned and calibrated, was completed on April 4, 1994. This verification period was totally successful with no major anomalies noted. Finally, the concept of on-orbit servicing was proven beyond the shadow of a doubt to be the way to keep the Hubble Observatory fully functional and performing cutting edge astronomy.

All pre-launch mission success criteria were fully met with the completion of all primary and secondary servicing tasks.

Based upon the completion of the pre-launch mission objectives and the meeting of all mission success criteria, it is judged that the STS-61 first HST Servicing mission was totally successful.

However, the overall success of HST, as a scientific program, must be judged on its performance over its planned 15-year lifetime, of which the first servicing mission was a overwhelmingly significant and important milestone.

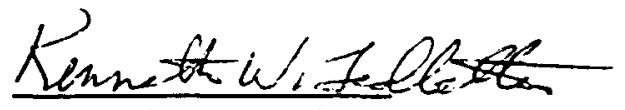

Kenneth $W$. Ledbetter

HST Program Manager

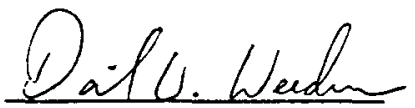

Daniel W. Weedman

Director, Astrophysics Division
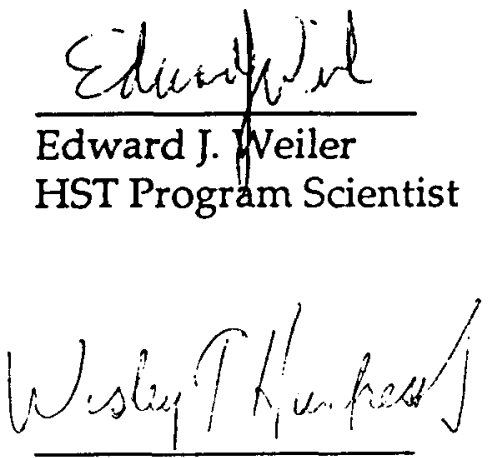

Wesley T. Huntress, Jr.

Associate Administrator for Space Science 